\title{
SATELLITE-SATELLITE LASER LINKS FOR FUTURE GRAVITY MISSIONS
}

\author{
P. L. BENDER (pbender@jila.colorado.edu), J. L. HALL \\ (jhallejila.colorado.edu) and J. YE (yedjila.colorado.edu) \\ JILA, National Institute of Standards and Technology and University of Colorado, Boulder, \\ Colorado 80309-0440 USA \\ W. M. KLIPSTEIN (klipsteinajp1.nasa.gov) \\ Jet Propulsion Laboratory, California Institute of Technology, Pasadena, California 91109, USA
}

Received: 15 May 2002; Accepted in final form: 15 August 2002

\begin{abstract}
A strong candidate for use in future missions to map time variations in the Earth's gravity field is laser heterodyne measurements between separate spacecraft. At the shortest wavelengths that can be measured in space, the main accuracy limitation for variations in the potential with latitude is expected to be the frequency stability of the laser. Thus the development of simple and reliable space-qualified lasers with high frequency stability appears to be an important goal for the near future.

In the last few years, quite high stability has been achieved by locking the second harmonic of a Nd:YAG laser to a resonant absorption line of iodine molecules in an absorption cell. Such a laser system can be made quite robust, and temperature related frequency shifts can be controlled at a low value. Recent results from laboratory systems are described. The Allan standard deviation for the beat between two such lasers was $2 \times 10^{-14}$ at $10 \mathrm{~s}$, and reached $7 \times 10^{-15}$ at $600 \mathrm{~s}$.
\end{abstract}

\section{Introduction}

The use of laser heterodyne measurements between separate spacecraft to map the Earth's gravity field and time variations in it has been discussed by a number of authors (see, e.g., Schumaker, 1990; Bender, 1992; Colombo and Chao, 1992; Watkins et al., 2000). As an example, the type of GRACE follow-on mission considered by Watkins et al. (2000) will be discussed briefly. To be specific, we assume the following: that the Allan standard deviation (fractional frequency fluctuation) for the laser frequency is $1 \times 10^{-14}$ from 10 to $5000 \mathrm{~s}$ period; that the spurious acceleration noise level for the test mass in the accelerometer on each spacecraft is $10^{-12} \mathrm{~m} / \mathrm{s}^{2} / \sqrt{\mathrm{Hz}}$ down to at least $0.001 \mathrm{~Hz}$; that the spacecraft separation is 100 $\mathrm{km}$; and that the altitude of the nearly polar orbit is about $300 \mathrm{~km}$. The assumed Allan standard deviation corresponds to a spectral amplitude of the fractional laser frequency variations of

$$
(\delta v) /(v)=\left(0.85 \times 10^{-14}\right)(1 \mathrm{~Hz} / f)^{0.5} / \sqrt{\mathrm{Hz}},
$$

where $f$ is the frequency of the variations. 
The lasers in the two spacecraft are assumed to be independently stabilized. The received beam from the other spacecraft is beat (heterodyned) against the local laser on each spacecraft, and the phase or frequency of the two resulting beat signals is measured as a function of time. Combining the results gives the biased range or integrated Doppler observable, and other observables can be obtained from this. The biased range is measured in terms of the laser wavelength, which provides the necessary scale factor. Thus fractional fluctuations in the laser frequency and wavelength translate directly into the same fractional fluctuations $\delta L$ in the measured range $L$.

Given Eq. (1) and the $100 \mathrm{~km}$ spacecraft separation in our example,

$$
\delta L=0.85 \times 10^{-9}(1 \mathrm{~Hz} / f)^{0.5} \mathrm{~m} / \sqrt{\mathrm{Hz}} .
$$

The corresponding acceleration noise $\delta a_{L}$ is obtained by multiplying by $(2 \pi f)^{2}$ :

$$
\delta a_{L}=3.4 \times 10^{-8}(f / 1 \mathrm{~Hz})^{1.5} \mathrm{~m} / \mathrm{s}^{2} / \sqrt{\mathrm{Hz}} .
$$

This is larger than $2^{0.5}$ times the assumed test mass acceleration noise for frequencies above $1.2 \times 10^{-3} \mathrm{~Hz}$, so the laser frequency noise dominates the assumed spurious acceleration noise for higher frequencies.

The distance measurement noise due to the laser frequency noise can be written as

$$
\delta L=2.7 \times 10^{-9}(0.1 \mathrm{~Hz} / f)^{0.5} \mathrm{~m} / \sqrt{\mathrm{Hz}},
$$

so it will be $2.7 \times 10^{-9} \mathrm{~m} / \sqrt{\mathrm{Hz}}$ or larger over the frequency range of 0.0002 to $0.1 \mathrm{~Hz}$ that is of interest for gravity field measurements in space. Since 2.5 or 3 times $10^{-9} \mathrm{~m} / \sqrt{\mathrm{Hz}}$ is about the satellite translational noise level $\delta L_{T}$ often quoted as expected for the drag-free systems planned for several other missions, it appears that the satellite-to-satellite distance measurements probably can be made between the optical benches on which the accelerometers for the drag-free systems are mounted. Alternately, measurements could be made directly between the test masses in the accelerometers, as is planned for the LISA mission (LISA Study Team, 2000).

Another possible error source for the distance measurements is the attitude variations of the satellite. The laser wavefronts transmitted from satellite A will be observed at satellite B as nearly spherical waves centered on the transmitting satellite. However, the deviations from spherical waves due to the laser beam and optical element imperfections will cause variations in the apparent distance when the attitude of satellite A changes. If the rms phase error over the transmitting aperture is $\lambda / 10$ and corresponds to pure astigmatism, and the transmitting aperture diameter is $\mathrm{D}$, the rms size of the resulting distance measurement error $\delta L_{a}$ at an angle $\theta$ to the axis is

$$
\delta L_{a}=[1 / 8 \sqrt{3}]\left[(\pi)^{2}\right][\lambda / 10]\left[(D / \lambda)^{2}\right]\left[(\theta)^{2}\right] .
$$


A result almost the same as this has been given by Robertson et al. (1997). The numerical coefficient is somewhat different, but only because of a different assumption about the shape of the transmitted wavefront error.

For $\lambda=1060 \mathrm{~nm}, D=2 \mathrm{~cm}$, a static offset error in the pointing of $\theta_{d c}$, and a pointing fluctuation $\delta \theta$,

$$
\delta L_{a}=54\left[\theta_{d c}\right][\delta \theta] \mathrm{m} .
$$

If $\theta_{d c}<2 \times 10^{-5} \mathrm{rad}$ and we require $\delta L_{a}<2.7 \times 10^{-9} \mathrm{~m} / \sqrt{\mathrm{Hz}}$, then the requirement on $\delta \theta$ is that it be less than $3 \times 10^{-6} \mathrm{rad} / \sqrt{\mathrm{Hz}}$.

The above pointing stability can be achieved fairly easily by using one of the approaches that have been developed for the LISA mission (Danzmann et al., 1998; Hammesfahr 2001). The laser beam from spacecraft A will be very bright at spacecraft $B$, and a portion of the received beam can be focused onto a CCD array with a fairly long effective focal length. Any change in the attitude of spacecraft $B$ can be detected as a motion of the spot on the CCD array. The motion can be used at medium and low frequencies to correct the spacecraft attitude, while fast motions can be handled by piezoelectrically controlled tilts of the transmitted laser beam.

With the above laser frequency, spacecraft translation, and beam pointing errors, the total errors near $0.1 \mathrm{~Hz}$ would be about

$$
\delta L=\left(5 \times 10^{-9}\right)\left[(0.1 \mathrm{~Hz} / f)^{0.5}\right] \mathrm{m} / \sqrt{\mathrm{Hz}},
$$

and

$$
\delta a=\left(2 \times 10^{-9}\right)\left[(f / 0.1 \mathrm{~Hz})^{1.5}\right] \mathrm{m} / \mathrm{s}^{2} / \sqrt{\mathrm{Hz}} .
$$

This "range acceleration" uncertainty is closely related to the gravity gradient uncertainty, for which the corresponding value is

$$
\delta \Gamma=\left(2 \times 10^{-14}\right)\left[(f / 0.1 \mathrm{~Hz})^{1.5}\right] / \mathrm{s}^{2} / \sqrt{\mathrm{Hz}},
$$

or

$$
\delta \Gamma=\left(2 \times 10^{-5}\right)\left[(f / 0.1 \mathrm{~Hz})^{1.5}\right] \text { Eötvös } / \sqrt{\mathrm{Hz}} .
$$

\section{Iodine Stabilized Laser}

There are many types of laser frequency stabilization schemes that could potentially be used in a space mission. However, one particularly attractive candidate for use in the near future is a Nd:YAG laser operating at $1064 \mathrm{~nm}$ wavelength, with its second harmonic stabilized to a molecular transition in iodine vapor. The NPRO type of Nd:YAG laser with output power levels of up to $200 \mathrm{~mW}$ has been spacequalified. With such a laser operating at $100 \mathrm{~mW}, 1$ or $2 \mathrm{~mW}$ of second harmonic 


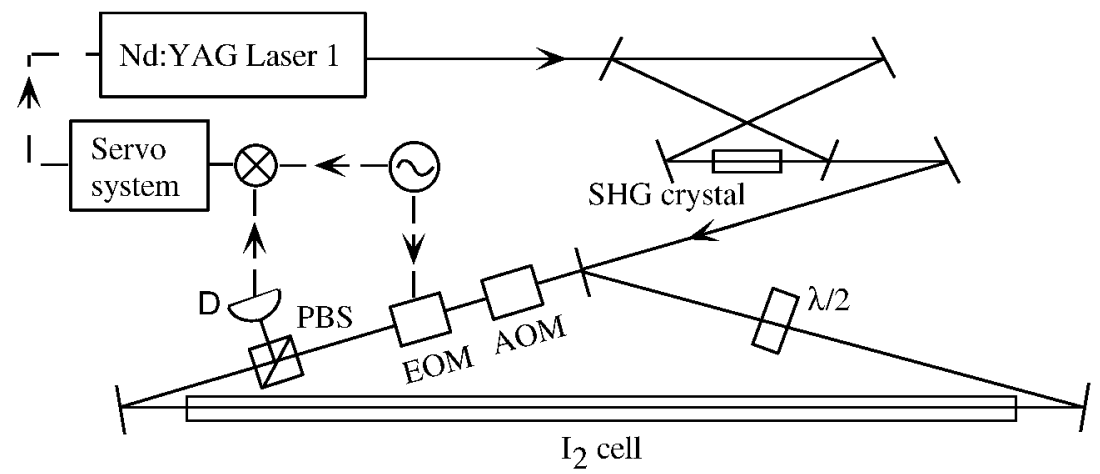

Figure 1. Diagram of a sub-Doppler iodine spectrometer: AOM, acousto-optic modulator; EOM, electro-optic modulator; PBS, polarization beam-splitter; D, detector; SHG, second-harmonic generation.

power can be generated with a periodically polled doubling crystal without requiring a power build-up cavity. This power level at $532 \mathrm{~nm}$ is sufficient for saturated absorption measurements in iodine vapor.

Measurements of the above type have been reported by Ye et al. $(1999,2001)$. Figure 1 shows a simple schematic of a sub-Doppler iodine spectrometer used for laser frequency stabilization. A saturating beam is sent in one direction through the absorption cell, and this causes a dip in the population difference between the lower and upper levels for the desired molecular transition. The width of the dip is determined by a combination of the natural linewidth, the collisional linewidth, the transit time broadening due to motion of the molecules across the laser beam, and some saturation broadening. However, the width of the dip will be considerably narrower than the full Doppler linewidth. For the $a_{10}$ hyperfine component of the $\mathrm{R}(56)$ 32-0 band, this width can be less than $1 \mathrm{MHz}$. A weaker probe beam is sent through the cell in the opposite direction, and is used to lock the laser frequency to that of the resulting narrow transmission peak. The $a_{10}$ hyperfine component is well separated from the other components of the line and is near the center of the overall hyperfine structure, so there is little effect from the wings of the other components.

The results of Ye et al. (2001) for the frequency stability of an iodine-stabilized laser are shown in Figure 2. The iodine vapor pressure was held at $0.8 \mathrm{~Pa}$ by cooling a side-tube to $-15^{\circ} \mathrm{C}$. For laser frequency stabilization work, the use of low sample pressure is important in terms of both minimizing the collision-induced pressure shift and reducing the influence on the baseline by the linear Doppler background. The signal size will of course decrease as the pressure goes down; however, the light path length inside the cell can be extended using a multi-pass strategy or even cavity enhancement. In the current work, the $1.2-\mathrm{m}$ cell length is already 
long enough to give adequate signal-to-noise ratio ( $\sim 120$ in a $10 \mathrm{kHz}$ bandwidth) without using a build-up cavity. Another important aspect of using lower pressure is that a lower optical power is needed to saturate the transition, because of the reduced collisional broadening. This yields a smaller power-related center frequency shift. The overall reduction of the operational resonance linewidth can to a certain degree compensate for the loss of the signal size since it is the ratio of the linewidth to the signal size that determines the residual rms frequency noise of the stabilized laser. The laser beams were collimated to give a $2 \mathrm{~mm}$ mode diameter in the center of the absorption cell, with the probe beam cross-section slightly smaller than the pump beam. With $1.0 \mathrm{~mW}$ of saturating power and $0.3 \mathrm{~mW}$ for the probe beam, a linewidth of $540 \mathrm{kHz}$ was achieved. The fractional frequency fluctuations (Allan standard deviation) achieved over measurement times $\tau$ are given as a function of $\tau$. The results can be described roughly as

$$
(\delta v / v)=7 \times 10^{-14} /\left[(\tau)^{0.5}\right]
$$

up to $100 \mathrm{~s}$ period, and constant between 100 and $600 \mathrm{~s}$. However, these results were obtained with a $1.2 \mathrm{~m}$ long absorption cell and an $I_{2}$ vapor pressure of about $15 \mathrm{~Pa}$ at $1{ }^{\circ} \mathrm{C}$.

For a space-qualified stable laser, limiting the absorption cell length to about $10 \mathrm{~cm}$ appears desirable. This can be done by putting the cell inside a bow-tie cavity, with a path length of about $50 \mathrm{~cm}$ and a finesse of roughly 35 , as discussed by Ishibashi, Ye and Hall (2002). Also, the $I_{2}$ vapor pressure would be reduced to about $0.8 \mathrm{~Pa}$ by operating with a side-tube temperature near $-15^{\circ} \mathrm{C}$. In this case, the pressure shift coefficient of $-3.2 \mathrm{kHz} / \mathrm{Pa}$ (Ye et al., 1999) and the temperature-dependent pressure shift slope of about $0.07 \mathrm{~Pa} / \mathrm{K}$ would give a nominal temperature coefficient of roughly $-220 \mathrm{~Hz} / \mathrm{K}$. However, some care in the temperature stabilization will be needed, since temperature changes for the rest of the cell can give transient $I_{2}$ pressure changes because of residual iodine molecules on the cell walls with different binding energies. But the more compact absorption cell reduces the temperature control problem considerably because of reducing the volume that has to be controlled.

With the type of system discussed above, the calculated frequency instability is about $3.6 \times 10^{-15} /\left(\tau^{0.5}\right)$ (Ishibashi et al., 2002). A possible alternate approach to using a low-finesse resonant cavity around the cell is to reduce the frequency noise in the NPRO laser below its usual level of about $5 \mathrm{kHz}$ at $1 \mathrm{~ms}$ by locking it to a stable resonant cavity for short times. However, until the performance of either type of system has been demonstrated with a space-qualifiable system, it appears desirable to limit our expectations for the performance of iodine-stabilized lasers in space in the near future to something like an Allan standard deviation of $1 \times 10^{-14}$ or better over almost all of the desired frequency band of 0.0002 to $0.1 \mathrm{~Hz}$, except possibly right at the lower end because of thermal effects at 1 cycle/rev.

Research directed toward the development of a space-qualifiable iodine-stabilized laser system currently is in progress at the Jet Propulsion Laboratory. Some 


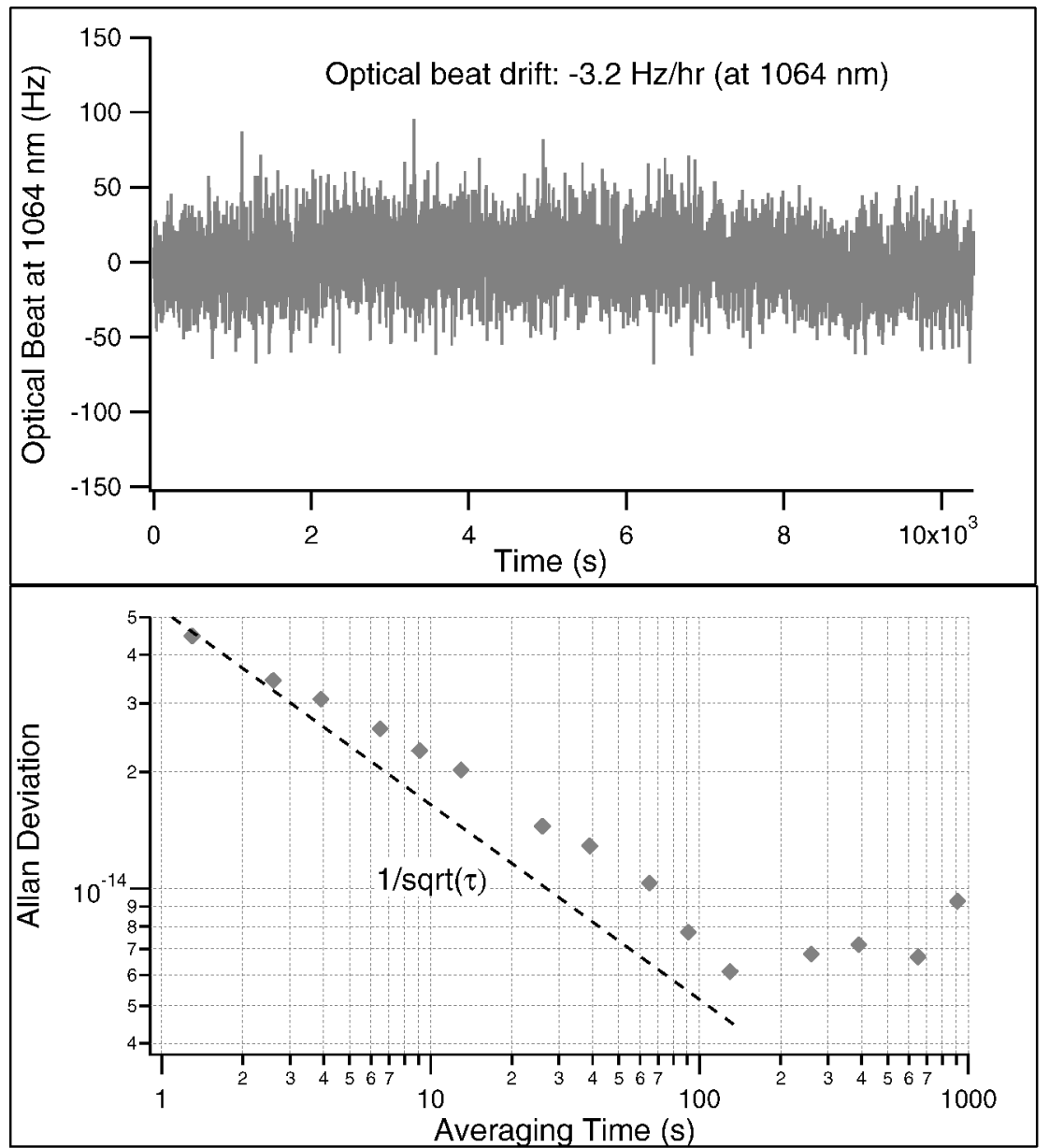

Figure 2. Time record of the beat frequency between the two iodine-stabilized Nd:YAG lasers, with a 1-s frequency-counting gate time. The Allan standard deviation is determined from the beat frequency data.

of the apparatus used in a preliminary laboratory system is shown in Figure 3. The length of the iodine cell shown in the lower part of the picture is $25 \mathrm{~cm}$.

To reach a higher frequency stability, it is useful to explore $I_{2}$ transitions with narrower transition linewidths. In fact, $I_{2}$ transitions near $514 \mathrm{~nm}$ have natural linewidths five times narrower than that at $532 \mathrm{~nm}$, while the signal-to-noise ratios of the recovered sub-Doppler signals are excellent. Recent results (Cheng et al., 2002) indicate that $I_{2}$ transitions at $514 \mathrm{~nm}$ hold a great promise for future development of high quality, portable optical frequency standards, especially considering the rapid development of all solid state Yb:YAG lasers around $1030 \mathrm{~nm}$. 


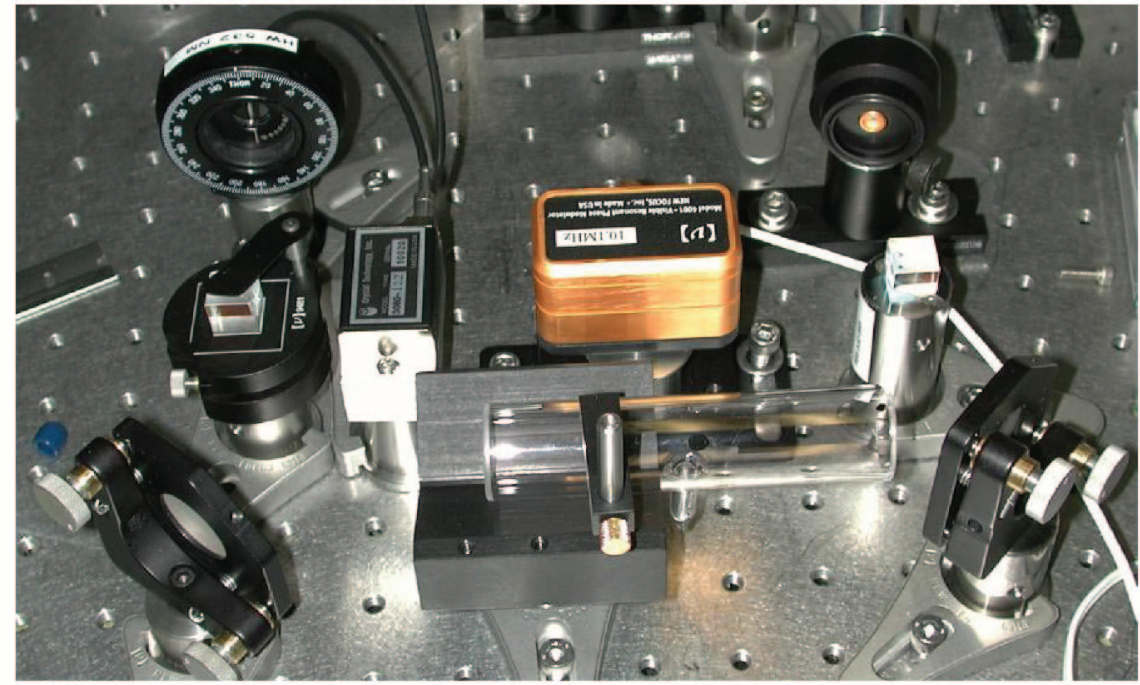

Figure 3. Preliminary laboratory system for use in research on iodine-stabilized lasers.

\section{Conclusions}

It appears practical to design and demonstrate iodine-stabilized lasers for use in space measurements of the Earth's gravity field in the near future. Suitable 1064 $\mathrm{nm}$ Nd:YAG lasers can be stabilized using only 1 or $2 \mathrm{~mW}$ of 2 nd harmonic power. The temperature control requirements can be simplified by using quite small iodine absorption cells and fairly low iodine vapor pressure. The expected fractional frequency stability is $1 \times 10^{-14}$ or better over almost all of the desired frequency range from 0.0002 to $0.1 \mathrm{~Hz}$.

There are substantial advantages to using a stabilized laser of this kind for measuring changes in distances between spacecraft rather than a microwave system. The iodine-stabilized laser system is considerably simpler than any microwave frequency sources of similar stability that have been developed so far. The transmission optics also are simpler for the lasers, and the effects of phase measurement noise are very small. Thus the laser heterodyne measurement systems can be quite compact, and put only modest constraints on the spacecraft design.

\section{Acknowledgements}

Part of the research described in this paper was carried out at the Jet Propulsion Laboratory, California Institute of Technology, under a contract with the National Aeronautics and Space Administration. 


\section{References}

Bender, P.L.: 1992, 'Integrated laser Doppler method for measuring planetary gravity fields,' in From Mars to Greenland: Charting Gravity with Space and Airborne Instruments, IAG Symposium, Vol. 110, Springer-Verlag, pp. 63-72.

Cheng, W.-Y., Chen, L., Yoon, T.-H., Hall, J. L., and Ye, J.: 2002, 'Sub-Doppler molecular iodine transitions near the dissociation limit ( 523 to $498 \mathrm{~nm}$ ), Opt. Lett. 827, p. 571.

Colombo, O.L. and Chao, B.F.: 1992, 'Global gravitational change from space in 2001,' IAG Symposium Vol. 112, Potsdam.

Danzmann, K.V. et al. : 1998, LISA-Laser Interferometer Space Antenna Pre-Phase A Report, 2nd edn., MPQ 233.

Hammesfahr, A.: 2001 'LISA mission study overview,' Class. \& Quantum Grav. 18, pp. 4045-4051.

Ishibashi, C., Ye, J. and Hall, J.L.: 2002, 'Issues and applications in ultra-sensitive molecular spectroscopy,' in Methods for Ultrasensitive Detection II, C. W. Wilkerson, Jr., Ed., SPIE Vol. 4634 pp. 58-69.

LISA Study Team: 2000, 'LISA Laser Interferometer Space Antenna: a Cornerstone Mission for the Observation of Gravitational Waves,' ESA-SCI(2000)11, European Space Agency.

Robertson, D. I., McNamara, P., Ward, H. and Hough, J.: 1997, 'Optics for LISA,' Class. \& Quantum Grav. 14, pp. 1575-1577.

Schumaker, B.L.: 1990, 'Scientific Applications of Frequency-Stabilized Laser Technology in Space, Jet Propulsion Laboratory Pub. 90-50, Caltech, Pasadena, CA, pp. 133-146.

Watkins, M.M., Folkner, W.M., Chao, B., and Tapley, B.D.: 2000, 'EX-5: A laser interferometer follow-on to the GRACE mission,' presented at GGG2000, Banff, Canada, 31 July - 5 August, 2000 (unpublished).

Ye, J., Robertson, L., Picard, S., Ma, L.-S., and Hall, J. L.: 1999, 'Absolute frequency atlas of molecular $I_{2}$ lines at $532 \mathrm{~nm}$,' IEEE Trans. Instrum. Meas. 48, pp. 544-549.

Ye, J., L.-S. Ma, and J. L. Hall: 2001, 'Molecular iodine clock'’ Phys. Rev. Lett. 87, pp. 270801/1-4.

Address for Offprints: Scientific Reports Office, JILA, 440 UCB, Boulder, Colorado 80309-0440

USA, sro@jila.colorado.edu 